\title{
AV timing in pacemaker patients with first-degree AV block: which is preferable, intrinsic $\mathrm{AV}$ conduction or pacing?
}

\author{
Yoshihiro Aizawa $^{1} \cdot$ Toshiko Nakai $^{1}$ [ $\cdot$ Yukitoshi Ikeya $^{1} \cdot$ Rikitake Kogawa $^{1} \cdot$ Yuki Saito ${ }^{1} \cdot$ Kazuto Toyama $^{1} \cdot$ Tetsuro Yumikura $^{1}$. \\ Naoto Otsuka ${ }^{1} \cdot$ Koichi Nagashima $^{1} \cdot$ Yasuo Okumura ${ }^{1}$
}

Received: 3 November 2021 / Accepted: 28 January 2022 / Published online: 8 February 2022

(c) The Author(s) 2022

\begin{abstract}
Some patients with pacemakers present with first-degree atrioventricular (AV) block. To avoid right ventricular (RV) pacing, preserving intrinsic $\mathrm{AV}$ conduction as much as possible is recommended. However, there is no clear cutoff $\mathrm{AV}$ interval to determine whether intrinsic $\mathrm{AV}$ conduction should be preserved or RV pacing should be delivered. This study aimed to compare a pacing mode-preserving, intrinsic $\mathrm{AV}$ conduction with the DDD mode delivering RV pacing in terms of echocardiographic parameters in patients with first-degree $\mathrm{AV}$ block and to investigate whether RV pacing induces heart failure (HF). Stroke volume (SV) was measured to determine the optimal AV delay with the intrinsic $\mathrm{AV}$ conduction rhythm and the DDD pacing delivering RV pacing. Echocardiographic evaluation was performed for 6-month follow-up period. Seventeen patients were studied. At baseline, mean intrinsic PQ interval was $250 \pm 40 \mathrm{~ms}$. SV was greater with $\mathrm{RV}$ pacing with optimal $\mathrm{AV}$ delay of $160 \mathrm{~ms}$ than with intrinsic $\mathrm{AV}$ conduction rhythm in all patients. Therefore, pacemakers were set to the DDD to deliver RV pacing. During follow-up, seven patients developed HF. Mean baseline E/E' ratio in patients who developed HF (HF group) during $\mathrm{RV}$ pacing was higher than in patients without $\mathrm{HF}$ (non $=\mathrm{HF}$ group; $17.9 \pm 8$ versus $11.5 \pm 2, P=0.018$ ) Even within $\mathrm{HF}$ group patients without a high baseline $\mathrm{E} / \mathrm{E}^{\prime}$ ratio, it increased with $\mathrm{RV}$ pacing $(22.2 \pm 6$ versus $11.6 \pm 2 ; P<0.001)$. In patients with pacemaker and first-degree AV block, RV pacing with the optimal AV delay of $160 \mathrm{~ms}$ increased SV. However, the risk of HF may be increased with $\mathrm{RV}$ pacing if the $\mathrm{E} / \mathrm{E}^{\prime}$ ratio is $>15$ during intrinsic $\mathrm{AV}$ conduction or $\mathrm{RV}$ pacing. $\mathrm{RV}$ pacing should be avoided in patients with high $\mathrm{E} / \mathrm{E}^{\prime}$ ratio under intrinsic $\mathrm{AV}$ conduction or $\mathrm{RV}$ pacing.
\end{abstract}

Keywords Long atrioventricular conduction $\cdot$ Right ventricular pacing $\cdot$ Heart failure $\cdot$ Optimal atrioventricular delay $\cdot$ Diastolic function

\section{Introduction}

Sick sinus syndrome and advanced atrioventricular (AV) block are often treated with a pacemaker. However, ventricular contraction during right ventricular (RV) pacing might be less efficient than physiological ventricular contraction during intrinsic $\mathrm{AV}$ conduction because of dyssynchrony caused by RV pacing [1-4]. Therefore, some studies have suggested that intrinsic AV conduction should be preserved to prioritize physiological ventricular contractions as much as possible [5-7]. Synchronization between the atria and ventricles is also important for cardiac function. In patients with functioning atria (without atrial fibrillation), ventricular contraction with appropriate timing after atrial contraction is an

Toshiko Nakai

nakai.toshiko@nihon-u.ac.jp

1 Department of Medicine, Division of Cardiology, Nihon University School of Medicine, 30-1 Ohyaguchi-kamicho, Itabashi-ku, Tokyo 173-8610, Japan important factor to ensure adequate stroke volume (SV). Although the intrinsic rhythm allows for physiological ventricular contraction, excessive delays in $\mathrm{AV}$ conduction time (PR interval) may lead to diastolic mitral regurgitation or adversely affect left ventricular (LV) function. It has been reported that echocardiography is useful for optimizing AV delay settings in patients with pacemakers [8]. AV synchronization is very important for maintaining adequate SV, particularly in patients with heart failure. Therefore, studies have been conducted to explore optimum AV delay for cardiac resynchronization therapy (CRT) [9-11].

Regarding pacemakers for bradycardia, some studies have reported on the optimization of AV delay [8-13]. However, it remains unknown whether intrinsic $\mathrm{AV}$ conduction with delays in $\mathrm{AV}$ conduction time exceeding physiological levels (corresponding to first-degree AV block) should be preserved or ventricular pacing with AV delay within physiological levels should be delivered to obtain better $\mathrm{LV}$ function. RV pacing may cause intraventricular conduction defects or dyssynchrony that leads to impaired LV function. Therefore, this study aimed to compare a pacing 
mode-preserving, intrinsic AV conduction with the DDD mode delivering $\mathrm{RV}$ pacing in terms of echocardiographic parameters in patients with first-degree AV block and to investigate whether $\mathrm{RV}$ pacing induces heart failure (HF).

\section{Materials and methods}

\section{Study design}

This prospective observation study was conducted in patients with pacemakers who had an intrinsic rhythm with electrocardiographic findings of first-degree AV block and regularly visited an outpatient clinic for pacemaker checks. The effects of intrinsic AV conduction rhythm with first-degree AV block in the AAI pacing mode and RV paced rhythm with optimal AV delay in the DDD pacing mode on SV were examined. The optimal AV delay to obtain maximum SV was determined under echocardiographic guidance. Patients were followed for 6 months after their pacemaker setting was changed from AAI to DDD pacing mode to determine whether they develop HF, while in the DDD pacing mode with optimal AV delay.

Eighteen patients who had first-degree AV block and without $\mathrm{RV}$ pacing (programming either AAI mode or preference intrinsic AV conduction mode) were enrolled in the study between July 2018 and September 2020. One patient was excluded, because atrial fibrillation was detected at screening and a total of 17 patients were studied. Table 1 shows baseline characteristics of enrolled patients. None of study patients had history of HF hospitalization. Two patients had HF symptoms, such as occasional shortness of breath with exercise, and were being treated with medication. The patients underwent blood sampling for $\mathrm{N}$-terminal pro-brain natriuretic peptide (NT-proBNP) testing. They also underwent transthoracic echocardiography. SV was measured (1) under intrinsic AV conduction (PQ interval $>200 \mathrm{~ms}$ ) and with AV delays of (2) $120 \mathrm{~ms}$, (3) $160 \mathrm{~ms}$, and (4) $200 \mathrm{~ms}$, respectively. The AV delay that provided the maximum SV was considered the optimal AV delay. At the start of the 6-month follow-up period, the pacemakers of all patients were set to DDD pacing mode with an optimal AV delay of $160 \mathrm{~ms}$. The patients were monitored for signs of HF, such as symptom of New York Heart Association class II or more, or pulmonary congestion or increasing cardiothoracic ratio on chest X-ray, and changes in NT-proBNP levels during the follow-up period. This study was approved by the ethics committee of Nihon University Itabashi Hospital. Written informed consent was obtained from all patients (approval number: RK-180313-10).

\section{Diagnosis of heart failure}

The definition of HF was based on the Framingham criteria [14]. The major criteria consisted of paroxysmal nocturnal dyspnea, orthopnea, abnormal jugular venous distention, pulmonary rales, cardiomegaly, pulmonary edema, presence of a third heart sound,
Table 1 Baseline patient characteristics

\begin{tabular}{ll}
\hline Age (years) & $78 \pm 9$ \\
Male gender, n (\%) & $6(35)$ \\
Pacemaker indication & \\
Sick sinus syndrome, $n(\%)$ & $12(70)$ \\
Transient or advanced atrioventricular block, $n(\%)$ & $5(30)$ \\
AV interval (ms) & $250 \pm 40$ \\
Intrinsic QRS duration (ms) & $115 \pm 25$ \\
Hypertension, $n(\%)$ & $12(71)$ \\
Diabetes mellitus, $n(\%)$ & $1(6)$ \\
Medication & \\
Beta-blocker, $n(\%)$ & $9(53)$ \\
ACEI/ARB, $n(\%)$ & $11(65)$ \\
Antiarrhythmic agent, $n(\%)$ & $4(24)$ \\
Echocardiographic assessment & \\
LVDd (mm) & $48 \pm 4$ \\
LVDs (mm) & $32 \pm 4$ \\
Ejection fraction (\%) & $63 \pm 6$ \\
Left atrial dimension (mm) & $40 \pm 8$ \\
\hline
\end{tabular}

$A C E I$ angiotensin-converting enzyme inhibitor, $A R B$ : angiotensin receptor blocker, $A V$ atrioventricular, $L V D d$ left ventricular diastolic diameter, $L V D s$ left ventricular systolic diameter

central venous pressure $>16 \mathrm{cmH}_{2} \mathrm{O}$, and hepatojugular reflex. The minor criteria consisted of edema, night cough, hepatomegaly, pleural effusion, tachycardia $>120 \mathrm{bpm}$, and weight loss $>4.5 \mathrm{~kg}$ in 5 days (considered a major criterion if it occurs during therapeutic interventions for $\mathrm{HF}$ ). A patient was considered to have $\mathrm{HF}$ if two major criteria were present or if one major and two minor criteria were present concurrently. Two cardiologists confirmed the findings in each patient.

\section{Echocardiographic measurements}

Left ventricular ejection fraction (LVEF) and LV mass was calculated with the formula derived from American Society of Echocardiography data [15].LVEF was generally calculated using the modified Quinones method [16], but the modified Simpson's method was used in patients with LV dysfunction [17]. LV mass index was calculated as the LV mass-to-body surface area ratio [18]. Transmitral flow velocity curves were recorded to measure peak early diastolic flow velocity $(\mathrm{E})$ and late diastolic flow velocity (A). Tissue Doppler imaging was performed at the level of the septal mitral annulus to measure early diastolic myocardial velocity ( $\mathrm{E}^{\prime}$ ) and late diastolic myocardial velocity (A'), as previously described. A parasternal long axis view was obtained. The diameter of left ventricular oufflow tract (LVOT) was measured during mid-systole. An apical five-chamber view was obtained. Left ventricular outflow tract velocity time integral (LVOT VTI) was measured using pulsed-wave Doppler in the LVOT using a $2 \mathrm{~mm}$ sample volume positioned just proximal to the aortic valve. LVOT VTI is considered to be a surrogate of SV according to the following equations: 


\section{Determination of the optimal AV interval in DDD pacing mode}

All patients presented with intrinsic AV conduction with a first-degree AV block. At first, echocardiographic assessment including SV was performed with the intrinsic AV conduction rhythm as the baseline. Next, the pacemaker was programmed to the DDD pacing mode while varying the AV delay interval to 120,160 , and $200 \mathrm{~ms}$ to determine which setting corresponds to maximum SV. The pacemaker was programmed to the AV delay interval of $160 \mathrm{~ms}$ with maximum SV. The setting continued for 6 months, unless cardiac events occurred.

\section{Statistical analysis}

Continuous variables are presented as means $\pm \mathrm{SD}$. Categorical variables are expressed as numbers and percentages. Differences between groups were assessed using Student's $t$ test for normally distributed continuous variables and the Mann-Whitney $U$ test for non-normally distributed continuous variables. Fisher's exact test or the chi-squared test was used for categorical variables. $P<0.05$ was considered to be significant.

\section{Results}

\section{Study patients}

Table 1 shows patient characteristics at baseline. The study included 17 patients with mean age of $78 \pm 9$ years. There were 6 males (35\%) and 11 females (65\%). The most common underlying heart disease was sick sinus syndrome $(n=12 / 17 ; 71 \%)$. Mean intrinsic PQ interval was $250 \pm 40 \mathrm{~ms}$ and mean intrinsic QRS duration was $115 \pm 25 \mathrm{~ms}$. Systolic function was preserved in all patients, and mean LVEF was $63 \pm 6 \%$. Median NT-proBNP level at screening was $257 \mathrm{pg} / \mathrm{dl}$ (interquartile range 105-665 pg/dl).

SV during RV pacing with the optimal AV delay of $160 \mathrm{~ms}$ was greater than $\mathrm{SV}$ during intrinsic rhythm in all patients $(59 \pm 12$ vs. $67 \pm 11 \mathrm{ml} ; P<0.001$; Fig. 1). Therefore, at the start of followup, pacemakers were set to deliver RV pacing in the DDD pacing mode. For patients who developed HF with RV pacing during the 6-month follow-up period, the pacemaker was re-programmed to the initial setting to preserve intrinsic AV conduction with firstdegree AV block whenever possible. We originally planned to enroll many more patients in this study. However, we terminated enrollment when the number of participants reached 17 , because the incidence of heart failure was higher than expected.

\section{Clinical follow-up}

During the 6-month follow-up period with RV pacing, seven patients developed or relapsed into acute decompensated HF. Patients who developed heart failure during the follow-up period were categorized into the heart failure (HF) group. Those who did not develop heart failure were classified into the non-heart failure (non-HF) group. When outcomes were compared, there were no significant differences in baseline electrocardiographic findings and mean QRS duration after the start of RV pacing between the groups (Table 2). Mean NT-proBNP in the HF group was significantly higher than in the non-HF group $(166 \pm 117$ vs. $958 \pm 740 \mathrm{pg} / \mathrm{dl} ; P=0.004)$. Although there was a significant difference in $\mathrm{EF}$ at the follow-up assessment between the non-HF and $\mathrm{HF}$ groups ( $66.2 \%$ versus $58.6 \%, P=0.0459)$, the $\mathrm{HF}$ group had relatively preserved $\mathrm{EF}$ (Fig. 2). The $\mathrm{E} / \mathrm{E}^{\prime}$ ratio was the only echocardiographic parameter that was significantly higher in the HF group in the AAI pacing mode for preserving intrinsic rhythm $(11.0 \pm 1.5$ vs. $17.9 \pm 8.2 ; P=0.018)$. Figure 3 shows changes in $\mathrm{E} / \mathrm{E}^{\prime}$ ratio from the intrinsic rhythm phase to the $\mathrm{RV}$ pacing phase in both groups. In the non-HF group, no significant changes in $\mathrm{E}^{\prime} \mathrm{E}^{\prime}$ ratio were observed during intrinsic rhythm or RV pacing. None of the patients had an $E / E^{\prime}$ ratio reaching $>15$. In the HF group, all patients had $\mathrm{E} / \mathrm{E}^{\prime}$ ratios $>15$ during $\mathrm{RV}$ pacing.

Pacemaker settings were re-programmed to prolong AV delay and preserve intrinsic rhythm in all patients in the HF group. However, patients 5, 6, and 10 still required $\mathrm{RV}$ pacing, because they did not have intrinsic QRS activity even though the AV delay was prolonged to the maximum possible level. All seven patients in the HF group improved clinically during hospitalization and continued treatment in the outpatient clinic. However, Patients 5 and 6 were re-admitted due to worsening heart failure within the follow-up period. One underwent CRT pacemaker (CRT-P) implantation to enhance treatment. In the non-HF group, no significant changes in $\mathrm{E} / \mathrm{E}^{\prime}$ ratio $(11.0 \pm 1.5$ vs. $11.6 \pm 2.1 ; P=0.44)$ or NT-proBNP levels ( $166 \pm 117$ vs. $153 \pm 112 \mathrm{pg} / \mathrm{ml} ; P=0.72$ ) were observed during the follow-up period.

\section{Discussion}

In this study, maximum SV was obtained during RV pacing with optimal AV delay rather than during intrinsic $\mathrm{AV}$ conduction in patients with pacemakers and first-degree AV block. However, even though the pacemaker was programmed to the optimal setting based on echocardiographic assessment, seven (41\%) patients developed HF, suggesting that some patients may have better hemodynamics in a pacing mode that preserves intrinsic ventricular contraction with very long $P R$ intervals than with $\mathrm{RV}$ pacing in the DDD pacing mode with an optimal AV delay, which is believed to be more physiological. This is the first study that compared the incidence of HF between a pacing mode-preserving intrinsic ventricular contraction with prolonged $\mathrm{PR}$ intervals and RV pacing with the optimal $\mathrm{AV}$ delay in patients with pacemakers.

Sweeney et al. reported that RV pacing induces HF and atrial fibrillation in the Mode Selection Trial (MOST) [9]. The development of HF in seven patients in the present study supports this report. On the other hand, Riahi et al. reported that RV pacing 
in the DDD pacing mode is not associated with the development of HF [19]. Ten patients in the present study did not develop HF, similar to the results from Riahi et al.

There are many studies indicating that RV pacing is harmful [5-7]. Therefore, pacemaker manufacturers have developed pacemakers with new algorithms that preserve intrinsic conduction. These algorithms include Managed Ventricular Pacing $\left(\mathrm{MVP}^{\mathrm{TM}}\right.$; Medtronic, Minneapolis, USA) and Ventricular Intrinsic Preference (VIP ${ }^{\mathrm{TM}}$; Abbott Laboratories, Illinois, USA). In our clinical practice, there have been questions about what is the maximum acceptable prolongation of the PR interval and whether intrinsic $\mathrm{AV}$ conduction should be preserved even if the PR interval is $\geq 300 \mathrm{~ms}$ when these algorithms are applied. In the present study, $\mathrm{RV}$ pacing did not lead to adverse events in approximately $60 \%$ of patients. However, RV pacing led to HF in approximately $40 \%$ of patients. These findings cannot completely suggest that $\mathrm{RV}$ pacing is harmful. In this study, some patients developed HF even though all patients had LVEF $>50 \%$ at baseline and their pacemakers were programed with the optimal $\mathrm{AV}$ delay to provide the maximum $\mathrm{SV}$, suggesting that the development of HF might not always be associated with impaired systolic function. In contrast, some studies reported that worsening of $\mathrm{HF}$ is associated with impaired systolic function [20, 21]. E/E' ratio has commonly been used as an index of diastolic function in clinical practice and research studies [22, 23]. The present study compared changes in $\mathrm{E} / \mathrm{E}^{\prime}$ ratio before and after RV pacing between the HF and non-HF groups. Compared with the non-HF group, E/E' ratio worsened to $>15$ or increased from baseline in the HF group. Therefore, patients with impaired diastolic function or who develop left heart strain during $\mathrm{RV}$ pacing might be at risk of developing $\mathrm{HF}$ associated with RV pacing. Although it is difficult to fully explain why the optimal AV delay sometimes leads to HF, we speculate that RV pacing can lead to systolic dyssynchrony despite AV delay optimization, resulting in diastolic dysfunction. In this study, optimal AV delay

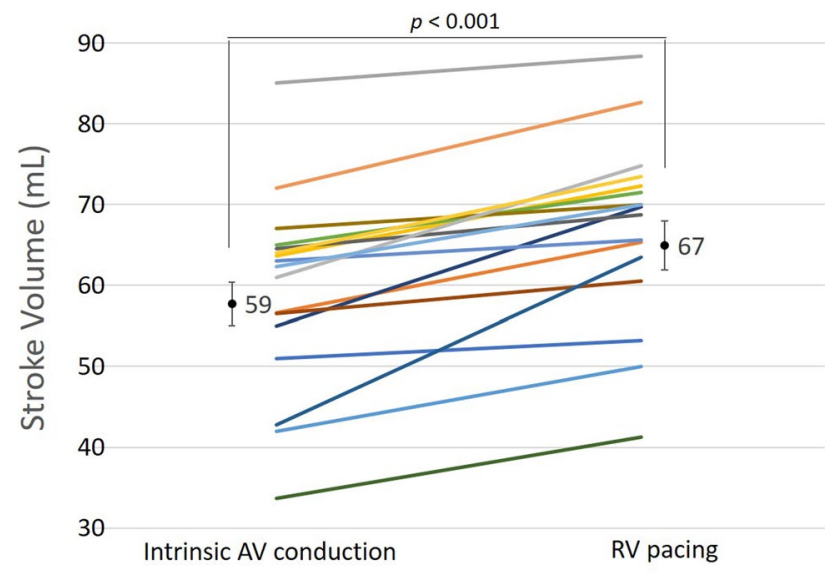

$\mathrm{AV}$, atrioventricular; RV, right ventricular

Fig. 1 Change in stroke volume with RV pacing. Stroke volume increased with RV pacing at optimal AV delay in all patients. $A V$ atrioventricular, $R V$ right ventricular was determined based on echocardiographic assessment. Echocardiography was performed in the resting state and this programming might not be the best in situations when heart rate increases. Intrinsic AV conduction might differ as heart rate increases. Therefore, it is possible that the optimal $\mathrm{AV}$ delay was no longer optimal when the patient is active or in an emotional state. We believe that this is a limitation of $\mathrm{AV}$ optimization based on echocardiography, unless the echocardiographic assessment was performed during exercise (e.g., with an ergometer).

\section{Clinical implications}

The results of this study suggested that $\mathrm{E} / \mathrm{E}^{\prime}$ ratio might be useful for predicting an increase in heart strain and the development of heart failure in association with RV pacing. Previous studies have found RV pacing unfavorable and recommended intrinsic conduction [5-7]. However, it is unknown whether a very long $\mathrm{AV}$ conduction time is acceptable. In this study, the DDD pacing mode with optimal AV delay resulted in greater SV than very long intrinsic $\mathrm{AV}$ conduction based on echocardiographic assessment. Approximately $60 \%$ of the study patients did not develop HF during RV pacing, suggesting that the DDD pacing mode with the optimal AV delay, which is believed to be physiological, might be better than intrinsic conduction if the intrinsic $\mathrm{AV}$ conduction time is too long. On the other hand, the remaining $40 \%$ patients developed HF during RV pacing, indicating that intrinsic conduction should be preferred over RV pacing in such patients even if their intrinsic $\mathrm{AV}$ conduction time is substantially longer than the optimal AV delay. These findings might be consistent with findings from the Biventricular versus Right Ventricular Pacing in Heart Failure Patients with Atrioventricular Block (BLOCK HF) trial [24], which showed that CRT should be selected for patients with low cardiac function $(\mathrm{LVEF}<50 \%)$. This study might help determine whether the current pacemaker should be replaced with CRT-P to enhance treatment. E/E' ratios before and after RV pacing may be useful information for determining whether intrinsic $\mathrm{AV}$ conduction should be preferred or the current pacemaker should be replaced with CRT-P early in patients with first-degree AV block who will inevitably require pacing.

\section{Limitations}

This study has some limitations. First, the sample size was small, with only 17 patients. We originally planned to include more patients, but had to discontinue patient enrollment, because some patients developed $\mathrm{HF}$ associated with RV pacing and there was a possible risk of causing a disadvantage to patients. Second, followup duration was too short to determine the effect of RV pacing on $\mathrm{HF}$, due to the same reason as for the first limitation. However, despite this short follow-up duration, a relatively high number of patients developed HF unexpectedly. This result demonstrated that undesirable effects are possible with $\mathrm{RV}$ pacing in certain patients. 
Table 2 Comparisons between the non-heart failure and heart failure groups

\begin{tabular}{llll}
\hline & Non-HF group $(n=10)$ & HF group $(n=7)$ & $P$ value \\
\hline Age (years) & $77 \pm 10$ & $78 \pm 8$ & 0.540 \\
Male gender, $n(\%)$ & $4(40)$ & $2(28)$ & 0.653 \\
PQ interval (ms) & $237 \pm 23$ & $267 \pm 53$ & 0.126 \\
QRS duration (ms) & $116 \pm 22$ & $114 \pm 32$ & 0.901 \\
Pacing QRS duration (ms) & $158 \pm 39$ & $141 \pm 6$ & 0.364 \\
NT-proBNP (pg/dl) & $166 \pm 117$ & $957 \pm 740$ & 0.004 \\
Stroke volume (ml) & $55 \pm 10$ & $62 \pm 13$ & 0.075 \\
Ejection fraction (\%) & $64 \pm 5$ & $62 \pm 7$ & 0.542 \\
E/E' & $11.0 \pm 1.5$ & $17.9 \pm 8.2$ & 0.018 \\
Left atrial dimension (mm) & $38 \pm 8$ & $44 \pm 6$ & 0.072 \\
LV mass index (g/m $\left.{ }^{2}\right)$ & $99 \pm 16$ & $120 \pm 26$ & 0.063 \\
LV relative wall thickness & $0.40 \pm 0.05$ & $0.40 \pm 0.08$ & 0.940 \\
\hline
\end{tabular}

$E / E^{\prime}$ ratio of peak mitral $\mathrm{E}$ wave velocity to peak early diastolic myocardial velocity at the septum based on tissue Doppler imaging, $H F$ heart failure, $N T$-proBNP $\mathrm{N}$-terminal pro-brain natriuretic peptide, $L V$ mass index: left ventricular mass index



$\mathrm{EF}$, ejection fraction; $\mathrm{HF}$, heart failure; $\mathrm{RV}$, right ventricular.

Fig. 2 EF with RV pacing at the follow-up echocardiographic assessment by heart failure status. There were no significant differences in EF between the two groups. $E F$ ejection fraction, $H F$ heart failure, $R V$ right ventricular 

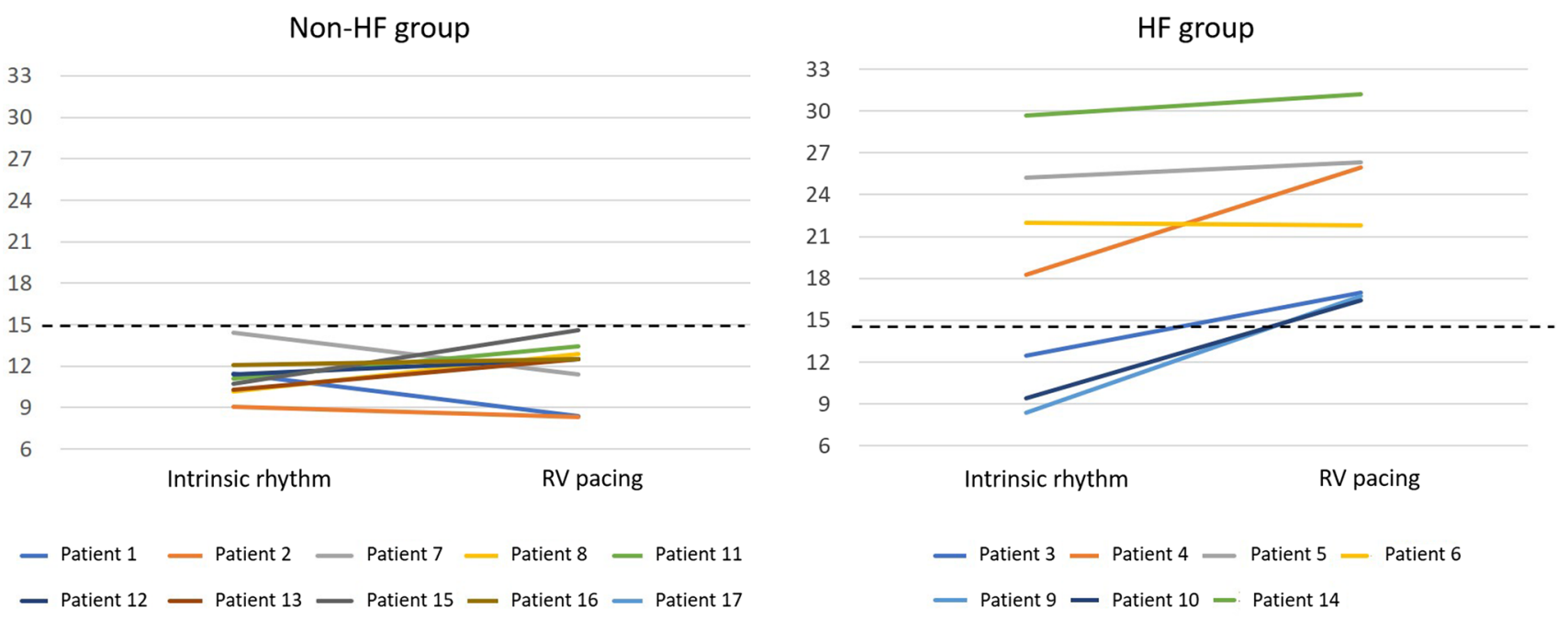

- Patient 1 - Patient $2-$ Patient 7 - Patient 8 - Patient 11
- Patient 12 Patient 13 Patient $15-$ Patient 16 - Patient 17

- Patient 9 - Patient 10 - Patient 14

$E / E^{\prime}$, ratio of peak mitral $E$ wave velocity to peak early diastolic myocardial velocity at the septum based on tissue Doppler imaging; $H F$, heart failure; RV, right ventricular.

Fig. 3 Change in $E / E^{\prime}$ ratio with RV pacing by heart failure status. Patients in Non-HF group showed low E/E' ratio both at baseline and after $\mathrm{RV}$ pacing. $E / E^{\prime}$ ratio of peak mitral $\mathrm{E}$ wave velocity to peak

Third, the AV delay that provided the maximum SV was selected as the optimal AV delay based only on echocardiography. We are not sure whether relying only on echocardiographic assessment is the gold standard. However, we selected this strategy because previous studies also set AV delay based on echocardiographic findings [8-13].

\section{Conclusions}

In patients with pacemakers and first-degree AV block, RV pacing with the optimal AV delay generally increased SV, but it should be used carefully because RV pacing may cause HF in some patients. $\mathrm{E} / \mathrm{E}^{\prime}$ ratio may be useful for predicting the development of $\mathrm{HF}$ associated with RV pacing.

Author contributions Conceptualization, YA and TN; methodology, KN; software, YS; validation, YI, RK and KT; formal analysis, NO; investigation, TY; data curation, $\mathrm{KN}$; writing — original draft preparation, YA; writing — review and editing, TN; visualization, KN; supervision, YO; All authors have read and agreed to the published version of the manuscript.

Funding This research did not receive any specific grant from funding agencies in the topic, commercial, or not-for-profit sectors.

Data availability The data in this study are available on request from the corresponding author. early diastolic myocardial velocity at the septum based on tissue Doppler imaging, $H F$ heart failure, $R V$ right ventricular

\section{Declarations}

Conflict of interest T.N. belongs to a department established by contributions from Abbot Medical, Biotronik Japan, Medtronic Japan, Japan Lifeline, and Boston Scientific. T.N. received lecture fees from Abbott Medical and Medtronic Japan. All other authors have no conflict of interest to report.

Ethical approval The study was conducted according to the guidelines of the Declaration of Helsinki, and approved by the Institutional Ethics Committee of Nihon University Hospital. (protocol code RK-180313-10, March 2016).

Informed consent A written informed consent has been obtained from the patients to publish this paper.

Open Access This article is licensed under a Creative Commons Attribution 4.0 International License, which permits use, sharing, adaptation, distribution and reproduction in any medium or format, as long as you give appropriate credit to the original author(s) and the source, provide a link to the Creative Commons licence, and indicate if changes were made. The images or other third party material in this article are included in the article's Creative Commons licence, unless indicated otherwise in a credit line to the material. If material is not included in the article's Creative Commons licence and your intended use is not permitted by statutory regulation or exceeds the permitted use, you will need to obtain permission directly from the copyright holder. To view a copy of this licence, visit http://creativecommons.org/licenses/by/4.0/.

\section{References}

1. Lee MA, Dae MW, Langberg JJ, Griffin JC, Chin MC, Finkbeiner WE, O'Connell JW, Botvinick E, Scheinman MM, Rosenqvist M (1994) Effects of long-term right ventricular apical pacing on left ventricular 
perfusion, innervation, function and histology. J Am Coll Cardiol 24(1):225-232

2. Zhang XH, Chen H, Siu CW, Yiu KH, Chan WS, Lee KL, Chan HW, Lee SW, Fu GS, Lau CP, Tse HF (2008) New-onset heart failure after permanent right ventricular apical pacing in patients with acquired high-grade atrioventricular block and normal left ventricular function. J Caldiovasc Electrophysiol 19(2):136-141

3. Thackray SDR, Witte KKA, Nikitin NP, Clark AL, Kaye GC, Cleland JGF (2003) The prevalence of heart failure and asymptomatic left ventricular systolic dysfunction in a typical regional pacemaker population. Eur Heart J 24:1143-1152

4. Ogano M, Tsuboi I, Iwasaki Y, Tanabe J, Shimizu W (2021) Structural heart disease, not the right ventricular pacing site, determines the QRS duration during right ventricular pacing. Heart Vessels 36:1870-1878

5. Chan WY, Blomqvist A, Melton IC, Norén K, Crozier IG, Benser ME, Eigler NL, Gutfinger D, Troughton RW (2014) Effects of AV delay and VV delay on left atrial pressure and waveform in ambulant heart failure patients: insights into crt optimization. Pacing Clin Electrophysiol 37(7):810-819

6. Fukuhara K, Okura H, Koyama T, Kume T, Neishi Y, Hayashida A, Yoshida K (2015) Feasibility of a novel atrioventricular delay optimization method using transmitral and pulmonary venous flow in patients with sequential ventricular pacing or cardiac resynchronization therapy. J Echocardiogr 13(2):52-58

7. Chatterjee NA, Gold MR, Waggoner AD, Picard MH, Stein KM, Yu Y, Meyer TE, Wold N, Ellenbogen KA, Singh JP (2016) Longer left ventricular electric delay reduces mitral regurgitation after cardiac resynchronization therapy: Mechanistic insights from the smart-av study (smartdelay determined av optimization: a comparison to other av delay methods used in cardiac resynchronization therapy). Circ Arrhythm Electrophysiol 9(11):e004346

8. Ishikawa T, Sumita S, Kimura K, Kikuchi M, Kosuge M, Kuji N, Endo T, Sugano T, Sigemasa T, Kobayashi I, Tochikubo O, Usui T (1999) Prediction of optimal atrioventricular delay in patients with implanted DDD pacemakers. Pacing Clin Electrophysiol 22(9):1365-1371

9. Sweeney MO, Hellkamp AS, Ellenbogen KA, Greenspon AJ, Freedman RA, Lee KL, Lamas GA (2003) Adverse effect of ventricular pacing on heart failure and atrial fibrillation among patients with normal baseline qrs duration in a clinical trial of pacemaker therapy for sinus node dysfunction. Circulation 107(23):2932-2937

10. Sharma AD, Rizo-Patron C, Hallstrom AP, O'Neill GP, Rothbart S, Martins JB, Roelke M,Steinberg JS, Greene HL, DAVID Investigators (2005) Percent right ventricular pacing predicts outcomes in the DAVID trial. Heart Rhythm 2(8):830-834

11. Sweeney MO, Bank AJ, Nsah E, Koullick M, Zeng QC, Hettrick D, Sheldon T, Lamas GA, Search AV Extension and Managed Ventricular Pacing for Promoting Atrioventricular Conduction (SAVE PACe) Trial (2007) Minimizing ventricular pacing to reduce atrial fibrillation in sinus-node disease. N Engl J Med 357(10):1000-1008

12. Ishikawa T, Sugano T, Sumita S, Nakagawa T, Hanada K, Kosuge M, Kobayashi I, Kimura K, Tochikubo O, Usui T, Umemura S (2001) Optimal atrioventricular delay setting determined by evoked QT interval in patients with implanted stimulus-T-driven DDDR pacemakers. Europace 3(1):46-51

13. Kato M, Dote K, Sasaki S, Goto K, Takemoto H, Habara S, Hasegawa D, Matsuda O (2005) Determination of the optimal atrioventricular interval in sick sinus syndrome during DDD pacing. Pacing Clin Electrophysiol 28(9):892-897

14. McKee PA, Castelli WP, McNamara PM, Kannel WB (1971) The natural history of congestive heart failure: the Framingham study. N Engl J Med 285:1441-1446
15. Takeda Y, Sakata Y, Higashimori M, Mano T, Nishio M, Ohtani T, Hori M, Masuyama T, Kaneko M, Yamamoto K (2009) Noninvasive assessment of wall distensibility with the evaluation of diastolic epicardial movement. J Card Fail 15(1):68-77

16. Quinones MA, Waggoner AD, Reduto LA, Nelson JG, Young JB, Winters WL Jr, Ribeiro LG, Miller RR (1981) A new, simplified and accurate method for determining ejection fraction with two-dimensional echocardiography. Circulation 64(4):744-753

17. Vuille C, Wayman AE (1994) Left ventricle I: general considerations, assessment of chamber size and function. In: Weyman AE (ed) Principles and practice of echocardiograpy, 2nd edn. Lea \& Febiger, Philadelphia, pp 575-624

18. Aizawa Y, Sakata Y, Mano T, Takeda Y, Ohtani T, Tamaki S, Omori Y, Tsukamoto Y, Hirayama A, Komuro I, Yamamoto K (2011) Transition from asymptomatic diastolic dysfunction to heart failure with preserved ejection fraction: roles of systolic function and ventricular distensibility. Circ J 75(3):596-602

19. RiahiS, Nielsen JC, Hjortshøj S, Thomsen PEB, Højberg S, Møller M, Dalsgaard D, Nielsen T, Asklund M, Friis EV, Christensen PerD, Simonsen EH, Eriksen UH, Jensen GVH, Svendsen JH, Toff WD, Healey JS, Andersen HR, DANPACE Investigators (2012) Heart failure in patients with sick sinus syndrome treated with single lead atrial or dualchamber pacing: no association with pacing mode or right ventricular pacing site. Europace 14:1475-1482

20. Redfield MM, Jacobsen SJ, Burnett JC, Mahoney DW, Bailey KR, Rodeheffer RJ (2003) Burden of systolic and diastolic ventricular dysfunction in the community: appreciating the scope of the heart failure epidemic. JAMA 289:194-202

21. Kane GC, Karon BL, Mahoney DW, Redfield MM, Roger VL, Burnett JC Jr, Jacobsen SJ, Rodeheffer RJ (2011) Progression of left ventricular diastolic dysfunction and risk of heart failure. JAMA 306:856-863

22. Nagueh SF, Appleton CP, Gillebert TC, Marino PN, Oh JK, Smiseth OA, Waggoner AD, Flachskampf FA, Pellikka PA, Evangelista A (2009) Recommendations for the evaluation of left ventricular diastolic function by echocardiography. J Am Soc Echocardiogr 22:107-133

23. McMurray JJV, Adamopoulos S, Anker SD, Auricchio A, Böhm M, Dickstein K, Falk V,Filippatos G, Fonseca C, Gomez-Sanchez MA, Jaarsma T, Køber L, Lip GYH, Maggioni AP, Parkhomenko A, Pieske BM, Popescu BA, Rønnevik PK, Rutten FH, Schwitter J, Seferovic P, Stepinska J, Trindade PT, Voors AA, Zannad F, Zeiher A, ESC Committee for Practice Guidelines (2012) ESC guidelines for the diagnosis and treatment of acute and chronic heart failure 2012: the task force for the diagnosis and treatment of acute and chronic heart failure 2012 of the European Society of Cardiology. Developed in collaboration with the Heart. Eur Heart J 33:1787-1847

24. Curtis AB, Worley SJ, Adamson PB, Chung ES, Niazi I, Sherfesee L, Shinn T, Sutton MS, Biventricular versus Right Ventricular Pacing in Heart Failure Patients with Atrioventricular Block (BLOCK HF) Trial Investigators (2013) Biventricular pacing for atrioventricular block and systolic dysfunction. N Engl J Med 368:1585-1593

Publisher's Note Springer Nature remains neutral with regard to jurisdictional claims in published maps and institutional affiliations. 